\title{
Factors Associated with Achievement of Inactive Disease in Children with Juvenile Idiopathic Arthritis Treated with Etanercept
}

\author{
NICOLETTA SOLARI, ELENA PALMISANI, ALESSANDRO CONSOLARO, ANGELA PISTORIO, STEFANIA VIOLA, \\ ANTONELLA BUONCOMPAGNI, MARCO GATTORNO, PAOLO PICCO, NICOLINO RUPERTO, \\ CLARA MALATTIA, ALBERTO MARTINI, and ANGELO RAVELLI
}

\begin{abstract}
Objective. To evaluate the rate of inactive disease in children with juvenile idiopathic arthritis (JIA) treated with etanercept, and to identify clinical characteristics associated with attainment of inactive disease.

Methods. Clinical charts of patients who were given etanercept between January 2002 and January 2011 were evaluated retrospectively. For each patient, all visits from initiation of etanercept to the last followup evaluation in which the patient was still receiving etanercept were examined to establish whether the patient had reached the state of inactive disease and to identify the first visit in which inactive disease was documented. Clinical characteristics associated with achievement of inactive disease were determined through univariate analyses and Cox regression procedures.

Results. A total of 173 patients who received etanercept for a median of 2.2 years (range $0.5-10.5$ yrs) were studied. Eighty-seven patients $(50.3 \%)$ achieved inactive disease after a median of 0.6 years (range 0.1-2.5 yrs) of therapy. At last followup evaluation, 85 patients $(49.1 \%)$ still had inactive disease and $70(40.5 \%)$ were in clinical remission on medication. The probability of achievement of inactive disease after 6,12, and 24 months of therapy was $24 \%, 46 \%$ and $57 \%$, respectively. On Cox regression analysis, the attainment of inactive disease was associated with lack of wrist involvement and an age at disease onset $<3.6$ years.

Conclusion. Around half of our patients with JIA treated with etanercept achieved a state of inactive disease. Children who lacked wrist involvement and were younger at disease onset had a greater likelihood of achieving inactive disease. (First Release Dec 1 2012; J Rheumatol 2013;40:192-200; doi:10.3899/jrheum.120842)
\end{abstract}

\section{Key Indexing Terms: \\ JUVENILE IDIOPATHIC ARTHRITIS OUTCOME PREDICTORS}

The advent of new therapies for juvenile idiopathic arthritis (JIA), particularly the introduction of biologic medications, has increased considerably the potential for treatment benefit, with clinical remission being now a realistic goal for a substantial proportion of patients $1,2,3,4$. These advances have led to a shift in the aim of therapy increasingly toward the attainment of complete disease quiescence $5,6,7,8,9,10$.

\footnotetext{
From the Istituto Giannina Gaslini and Università degli Studi di Genova, Genoa, Italy.

Supported in part by an educational grant from Pfizer (formerly Wyeth).

N. Solari, MD, Research Fellow; E. Palmisani, MD, Research Fellow; A. Consolaro, MD, Research Fellow; A. Pistorio, MD, PhD, Dirigente Medico; S. Viola, MD, Dirigente Medico; A. Buoncompagni, MD, Dirigente Medico; M. Gattorno, MD, Dirigente Medico; P. Picco, MD, Dirigente Medico; N. Ruperto, MD, MPH, Dirigente Medico, Istituto Giannina Gaslini; C. Malattia, MD, PhD, Assistant Professor; A. Martini, MD, Professor of Pediatrics; A. Ravelli, MD, Associate Professor of Pediatrics, Istituto Giannina Gaslini and Università degli Studi di Genova.

Address correspondence to Dr. A. Ravelli, Pediatria II, Istituto

G. Gaslini, Largo G. Gaslini 5, 16147 Genoa, Italy.

E-mail: angeloravelli@ospedale-gaslini.ge.it

Accepted for publication October 9, 2012.
}

\section{ETANERCEPT REMISSION}

Further, they have led to the view that in chronic arthritis clinical trials, it is important not only to know the magnitude of clinical improvement from baseline, but also to understand whether the therapeutic agent under study is able to achieve more pronounced levels of improvement, including a state of inactive disease 8,10 .

However, none of the registration trials of biologic agents in JIA to date has included inactive disease as primary endpoint. The preliminary definition of inactive disease in JIA $^{5,6}$ has been used as a primary outcome measure in a randomized, double-blind, placebo-controlled trial of 2 aggressive treatment strategies in children with early JIA ${ }^{11}$. Nevertheless, to gain further insight into the therapeutic efficacy of biologic medications there is a need to obtain information about their potential to induce clinical remission in standard clinical practice. Further, it would be desirable to identify factors associated with therapeutic response or nonresponse, to optimize the current therapeutic approaches.

Etanercept, a tumor necrosis factor- $\alpha(\mathrm{TNF}-\alpha)$ antagonist, has been the first biologic agent registered for use in

Personal non-commercial use only. The Journal of Rheumatology Copyright (c) 2013. All rights reserved. 
children with JIA. The efficacy and safety of this medication have been established in a randomized placebo-controlled withdrawal trial in patients with a polyarticular disease course who were refractory or intolerant to methotrexate ${ }^{12}$. Longterm extension studies of the original trial cohort and several national registries have subsequently confirmed the sustained clinical benefit and acceptable safety profile of the drug $13,14,15$. The evidence for the effectiveness of etanercept in JIA has been expanded by the observation that its administration may be associated with improvement of functional ability and quality of life ${ }^{16,17}$, recovery of growth velocity and bone status ${ }^{18,19}$, and reduction in the progression of radiographic joint damage ${ }^{20}$. However, the potential of etanercept to induce disease remission and the clinical features associated with treatment effectiveness have seldom been investigated ${ }^{21,22}$.

The primary objective of our study was 2-fold: first, to evaluate the proportion of children with JIA treated with etanercept who reached the state of inactive disease; and second, to identify clinical characteristics associated with attainment of inactive disease.

\section{MATERIALS AND METHODS}

Study design and patient selection. All consecutive patients who met the International League for Associations of Rheumatology (ILAR) criteria for $\mathrm{JIA}^{23}$ who were given etanercept at the study center between January 2002 and January 2011, and who had a minimum followup of 6 months after start of etanercept, were included in the study. The analysis was conducted through retrospective review of patient clinical charts and data stored in clinical databases. Patient information was collected by means of standardized case report forms and was entered in a specialized database. The study protocol was approved by the medical ethics committee at the Istituto G. Gaslini, Genoa, Italy.

Protocol of etanercept administration. All patients received etanercept subcutaneously at a dose of $0.8 \mathrm{mg} / \mathrm{kg} /$ week (maximum $50 \mathrm{mg}$ ). In the earlier years, the weekly dose was fractioned in 2 weekly administrations of $0.4 \mathrm{mg} / \mathrm{kg}$ (maximum $25 \mathrm{mg}$ ) in all patients. More recently, the weekly dose was mostly delivered in a single administration, using 25 or $50 \mathrm{mg}$ vials, depending on child's weight. During etanercept therapy, patients were evaluated clinically every 3 to 6 months. Laboratory monitoring was carried out every 8-12 weeks.

Assessment of inactive disease. For each patient, all visits from the start of etanercept therapy to the last followup evaluation in which the patient was still receiving etanercept were examined to establish whether the patient had achieved the state of inactive disease. In case the attending physician had started to decrease the weekly dosage or space dosing further apart before the last followup visit because of achievement of inactive disease, the last observation in which the patient was still receiving the standard dose of $0.8 \mathrm{mg} / \mathrm{kg} /$ week was retained as the last followup visit. In patients who achieved inactive disease, the first visit in which inactive disease was documented was identified. The state of inactive disease was defined, according to Wallace criteria ${ }^{5}$, as no joint with active arthritis, no systemic manifestations attributable to JIA, no active uveitis, normal acute-phase reactants, and physician's global assessment of disease activity indicating no disease activity (defined as score of 0 on a $0-10$ visual analog scale). The state of clinical remission on medication was assessed in all patients with inactive disease at last followup visit and was defined as a period of 6 continuous months of inactive disease while the patient was still receiving etanercept ${ }^{5}$.

Assessment of predictive factors. The following independent (predictor) variables were recorded: sex, age at disease onset, age and disease duration at treatment baseline, interval between first observation at study center and start of etanercept, ILAR category, antinuclear antibody (ANA) status (defined as reported ${ }^{24}$ ), JIA outcome measures at start of etanercept (Table 1), joints affected before start of etanercept, and medications administered before start of etanercept and administered concomitantly during etanercept therapy, including intraarticular corticosteroid injections.

Statistics. Descriptive statistics were reported as medians and interquartile ranges for continuous variables and as absolute frequencies and percentages for categorical variables. Comparisons between patients who did or did not achieve inactive disease were by Mann-Whitney $U$ test in case of quantitative data and chi-square test or Fisher's exact test, as appropriate, for categorical data.

Predictive factors were tested for association with the achievement of inactive disease during the time of observation. For each category of predictive factor tested, the number of patients with inactive disease, the time of observation (person-years), and the incidence rate of inactive disease per 100 person-years were calculated and compared by means of bivariate analyses. Factors significantly associated with inactive disease were then tested in a Cox proportional hazards regression model. The log-rank test was used for comparisons. Survival analysis, with the state of inactive disease as the event of interest, was conducted by Kaplan-Meier method $^{25}$. Survival curves were compared by the log-rank test.

The statistical packages used were Statistica (version 9.0, StatSoft Corp.) for bivariate analyses and Stata (release 7, Stata Corp.) for multivariate analyses.

\section{RESULTS}

Patient characteristics. A total of 187 patients were treated with etanercept in the study period. Fourteen patients were excluded from the analysis because the clinical chart could not be retrieved or the followup period after start of etanercept was shorter than 6 months. The main demographic and clinical features of the remaining 173 patients are presented in Table 1. Patients had on average a longer disease duration (median $5 \mathrm{yrs}$ ), although the median time interval between first observation and etanercept start was 3 years. The total duration of etanercept therapy was 458.8 patient-years, with a median treatment duration of 2.2 years per patient. The total number of clinic visits during etanercept therapy was 1142 , with a median of 5 visits per patient. The most frequent ILAR category was extended oligoarthritis, followed by rheumatoid factor-negative polyarthritis; $15.6 \%$ of patients had systemic arthritis. Around two-thirds of patients were ANA-positive. The median number of active joints at start of etanercept was 5 . The knee and ankle were the most frequently affected joints, followed by the hand and wrist joints.

Before start of etanercept, almost all patients had received methotrexate, $72.3 \%$ of patients had undergone intraarticular corticosteroid injections, and $40.5 \%$ of patients had received systemic corticosteroids. Concomitant medications administered during etanercept therapy included methotrexate in $67.1 \%$ of patients, intraarticular corticosteroids in $22 \%$, and systemic corticosteroids in $13.9 \%$.

Frequency of achievement of inactive disease. Eighty-seven patients (50.3\%) achieved inactive disease a median of 0.6 Personal non-commercial use only. The Journal of Rheumatology Copyright (C) 2013. All rights reserved. 
Table 1. Demographic, clinical, and therapeutic characteristics of patients with juvenile idiopathic arthritis treated with etanercept. Data are numbers (percentages) unless otherwise indicated.

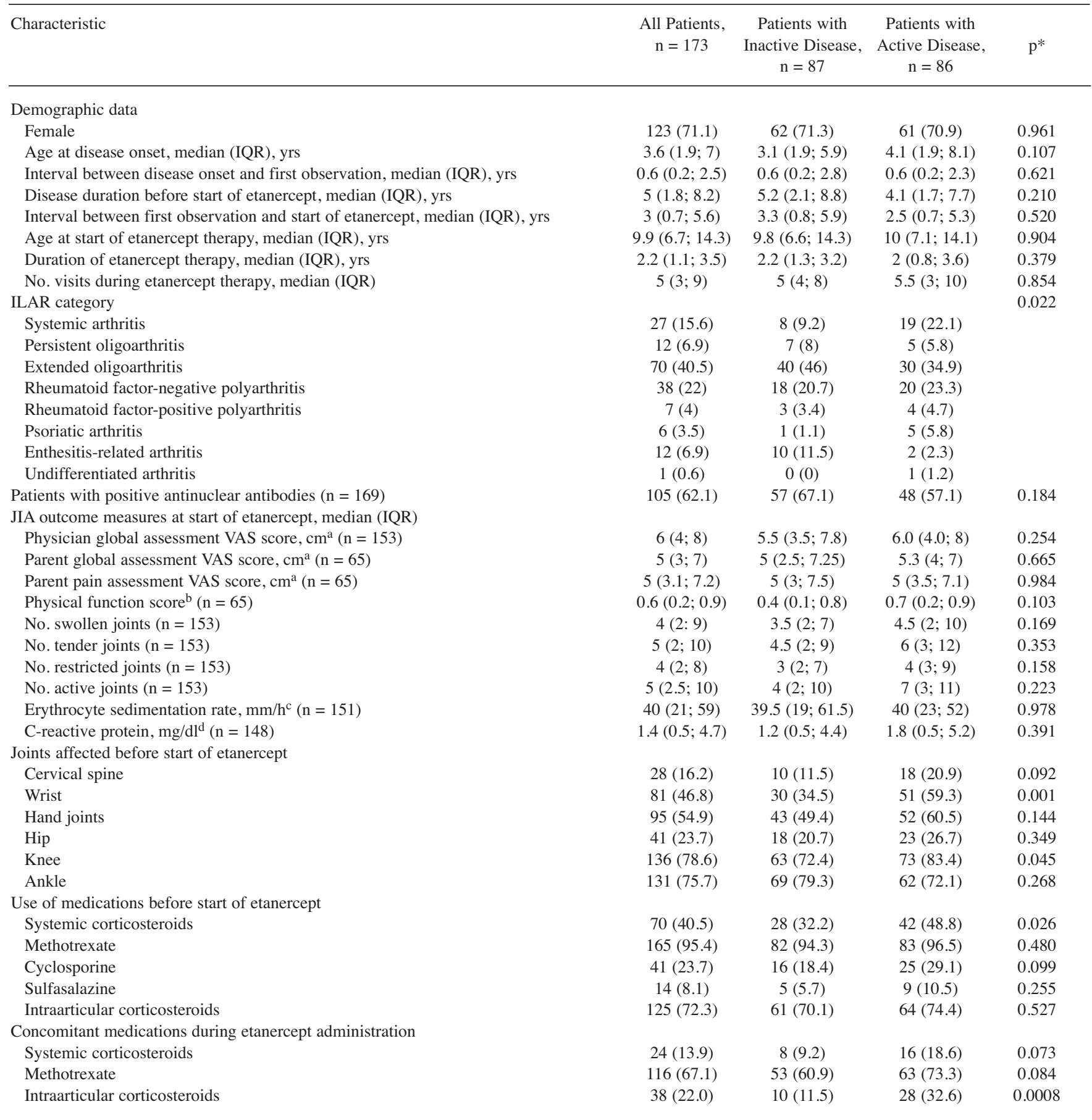

${ }^{\text {a }} 0-10$ scale $(0=$ best; $10=$ worst $) ;{ }^{b} 0-3$ scale $(0=$ best; $3=$ worst $) ;{ }^{\mathrm{c}}$ normal $<20 \mathrm{~mm} / \mathrm{h} ;{ }^{\mathrm{d}}$ normal $<0.3 \mathrm{mg} / \mathrm{dl}$. $*$ Comparisons of quantitative data by Mann-Whitney U test; comparisons of frequencies by chi-square test or Fisher's exact test if expected frequencies were < 5. IQR: interquartile range; ILAR: International League of Associations for Rheumatology; JIA: juvenile idiopathic arthritis; VAS: visual analog scale.

years (range 0.1-2.5 yrs) after initiation of etanercept therapy. The rate of inactive disease was much lower in children with systemic arthritis than in those with nonsystemic categories altogether ( $29.6 \%$ vs $54.1 \%$, respectively).
At last followup visit, 0.5 to 10.5 years after etanercept start (median 2.2 yrs), 85 patients (49.1\%) still had inactive disease and $70(40.5 \%)$ met the criteria for clinical remission on medication. The probability of achievement of 
inactive disease after 6,12 , and 24 months of therapy was $24 \%, 46 \%$, and $57 \%$, respectively.

Comparison of clinical characteristics between patients with and without inactive disease. Characteristics of patients who achieved or did not achieve inactive disease are compared in Table 1. Patients with inactive disease belonged more frequently to the extended oligoarthritis and enthesitis-related arthritis categories and less frequently to the systemic arthritis category than did patients without inactive disease. Further, patients with inactive disease had a lower frequency of wrist involvement at the start of etanercept, had less often received systemic corticosteroids before starting etanercept, and had more frequently undergone intraarticular corticosteroid injections during etanercept administration than did patients without inactive disease. The same comparison was made after the exclusion of the 27 children with systemic arthritis. In this analysis, the differences regarding ILAR category, corticosteroid therapy before start of etanercept, and intraarticular corticosteroid injections during etanercept administration were no longer detected, whereas patients with inactive disease still had a lower frequency of wrist involvement at start of etanercept than patients without inactive disease $(30.4 \%$ and $50.7 \%$, respectively; $\mathrm{p}=0.012$ ). Unlike the observations from the entire sample, patients with inactive disease had a younger age at disease onset than patients without inactive disease (median ages 2.8 yrs and 4.5 yrs, respectively; $\mathrm{p}=$ 0.046). Among the 27 patients with systemic arthritis, those with inactive disease $(n=8)$ had a longer disease duration than those without inactive disease $(n=19)$, with median disease durations of 6.6 years and 2.1 years, respectively ( $p=0.003)$, and they were older at etanercept initiation, with median ages of 11.8 years and 7.3 years, respectively $(\mathrm{p}=0.029)$.

Factors associated with achievement of inactive disease. For each patient and category of predictive factors tested for their association with inactive disease, Table 2 presents the number of patients in the predictive factor category out of the total number of patients with inactive disease, the time to inactive disease expressed in terms of person-years, and the incidence rate of inactive disease. Factors associated with achievement of inactive disease were age at disease onset younger than 3.6 years, a physician global rating $<6$, the absence of wrist disease, the lack of use of systemic corticosteroids before the start of etanercept, and the lack of administration of intraarticular corticosteroids during etanercept therapy. When all these variables were included in a Cox proportional hazards regression model, the age at disease onset younger than 3.6 years and the absence of wrist disease were the only variables associated with the attainment of inactive disease (Table 3). A secondary analysis after the exclusion of the 27 children with systemic arthritis identified the same predictors (Table 3 ).

The survival analysis, with the state of inactive disease as the event of interest, in the entire patient sample and in patients who did or did not have the 2 risk factors that were significantly associated with inactive disease in the Cox regression model is presented in Figures 1, 2, and 3, respectively.

Drug discontinuation and adverse events. After the last followup evaluation, 137 patients were maintained on etanercept therapy, whereas 36 patients were discontinued. The reasons for discontinuation were inefficacy (14 patients), remission (11), side effects (10), and lack of compliance (1 patient). Adverse events observed during etanercept administration included new-onset iridocyclitis (6 patients), leukopenia (3), thrombocytopenia (1), injection-site reactions (2), urticaria/angioedema (3), and papilledema (1 patient). Serious infections included varicella complicated by bronchopneumonia (1 patient) and tuberculosis (1 patient); 1 patient died of a streptococcal sepsis.

\section{DISCUSSION}

We evaluated the frequency of achievement of inactive disease in 173 children with JIA treated with etanercept in standard clinical care. We found that $50.3 \%$ of patients achieved inactive disease a median of 0.6 years after the initiation of etanercept therapy. At last followup visit, after a median of 2.2 years from start of etanercept, $49.1 \%$ of patients still had inactive disease and $40.5 \%$ met the criteria for clinical remission on medication (i.e., they had been in the state of inactive disease for at least 6 months while taking etanercept). The probability of achieving the inactive disease state after 6,12 , and 24 months of therapy was $24 \%$, $46 \%$, and $57 \%$, respectively.

The prevalence of inactive disease in JIA patients treated with etanercept was evaluated in 2 previous studies, both based on national registries. In the German registry, $47.6 \%$ and $26.6 \%$ of 787 patients reached the criteria for inactive disease or clinical remission on medication, respectively ${ }^{21}$. Among 262 patients included in the Dutch registry, the frequency of excellent response, defined as fulfillment of adapted inactive disease criteria, was $32 \%$ after 15 months of therapy. This rate increased to $37 \%-49 \%$ in a secondary longer-term followup analysis, 4 to 7 years after initiation of etanercept ${ }^{22}$. Together, the results reported in national registries and those from our study indicate that around half of children with JIA who are treated with etanercept in real-life clinical settings are able to attain complete disease quiescence.

In our study, the lack of involvement of the wrist joint was the clinical characteristic most strongly associated with achievement of inactive disease in Cox regression analysis. This observation implies that wrist disease is a marker of poorer therapeutic outcome in children with JIA treated with etanercept. Previous studies have shown that JIA patients with wrist disease are at high risk of developing structural joint damage $26,27,28$, a more severe course of arthritis ${ }^{29,30}$, or

Personal non-commercial use only. The Journal of Rheumatology Copyright (c) 2013. All rights reserved. 
Table 2. Patient and disease characteristics tested for association with achievement of inactive disease state.

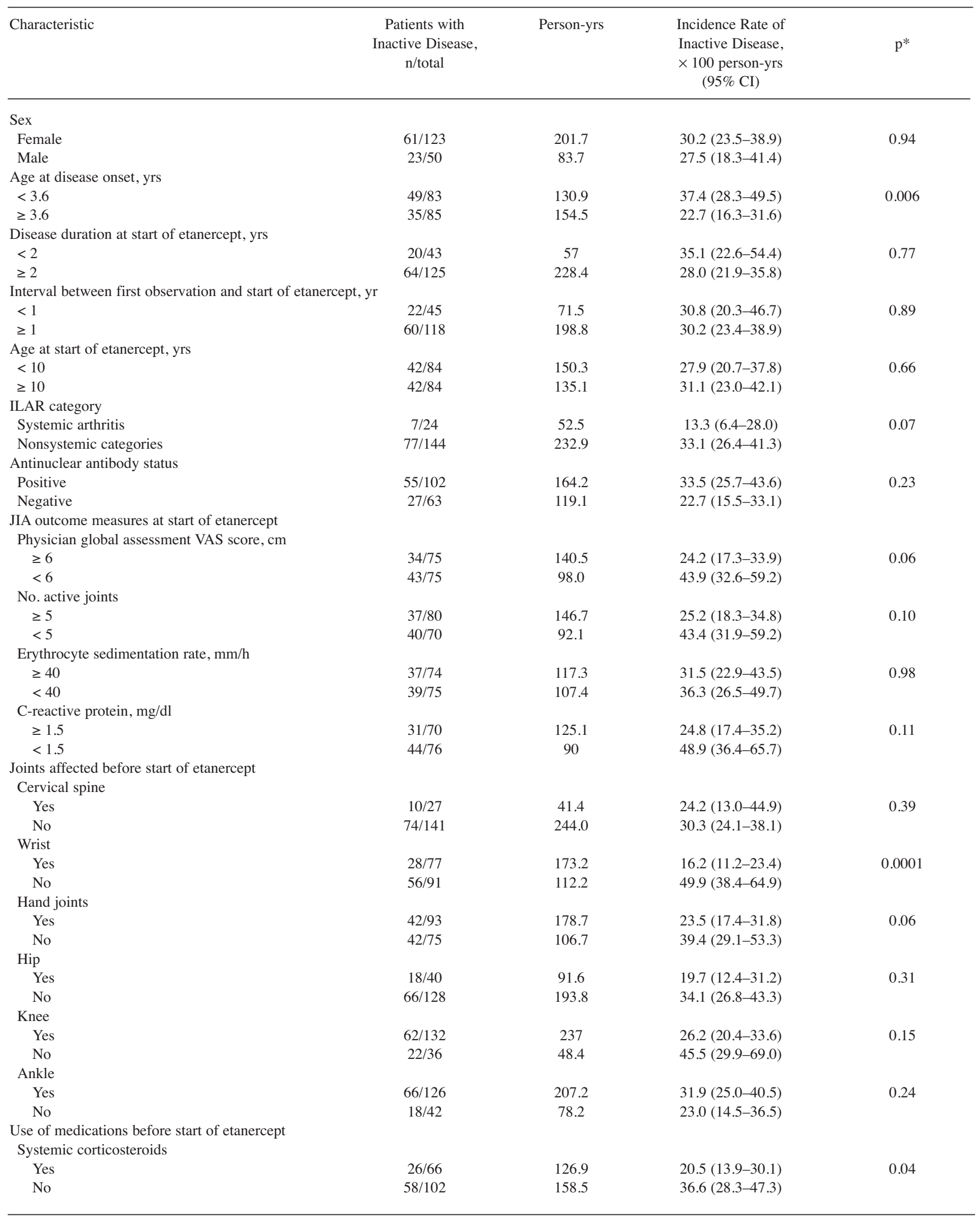




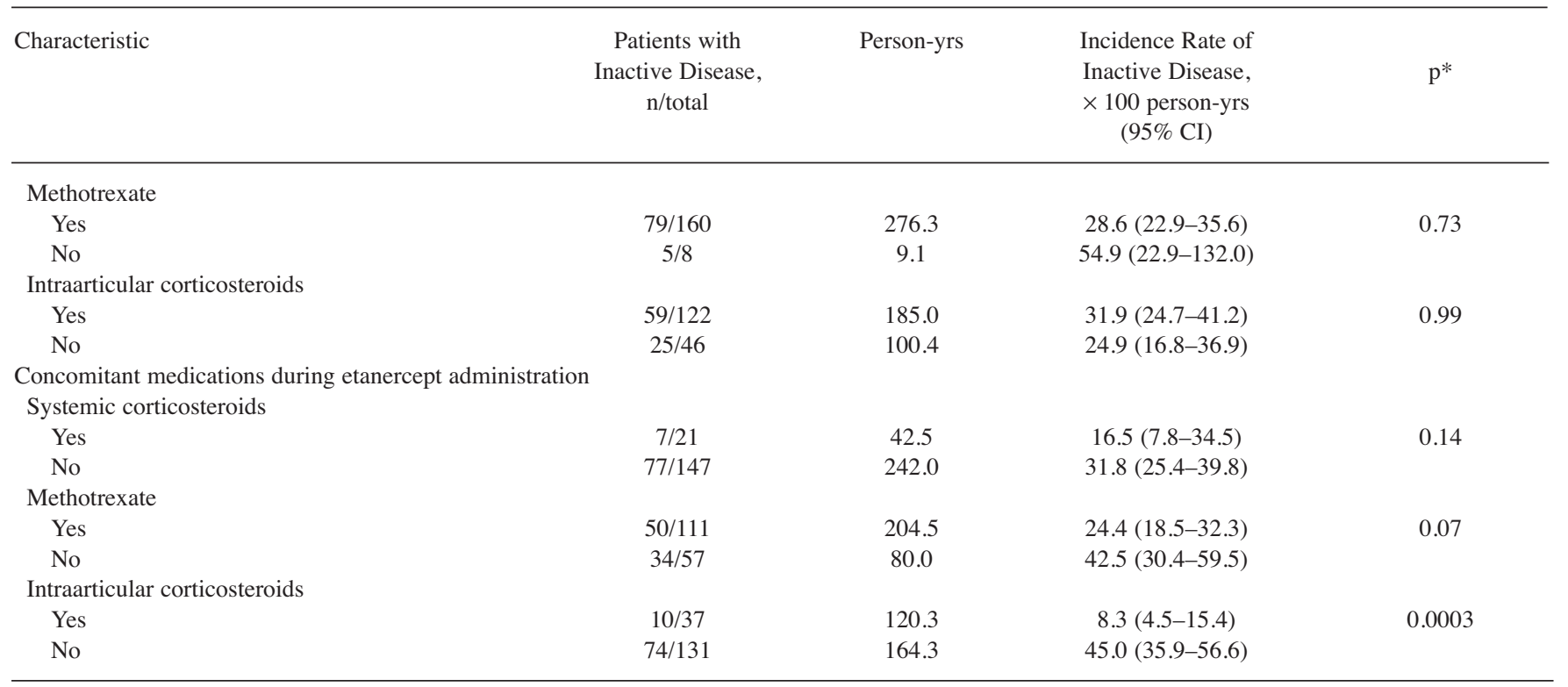

* Log-rank test. ILAR: International League of Associations for Rheumatology; JIA: juvenile idiopathic arthritis.

Table 3. Best-fitting model obtained through Cox proportional hazards regression procedures in all patients and in patients who did not have systemic arthritis. Achievement of inactive disease status was the dependent variable.

\begin{tabular}{lcc}
\hline Explanatory Variables & $\mathrm{HR}_{\mathrm{adj}}(95 \% \mathrm{CI})$ & $\mathrm{p}^{*}$ \\
\hline All patients, $\mathrm{n}=168$ & & \\
$\quad$ Absence of wrist involvement & $2.19(1.38-3.48)$ & 0.0006 \\
Age at disease onset $<3.6$ yrs & $1.61(1.04-2.49)$ & 0.03 \\
Patients without systemic arthritis, $\mathrm{n}=144$ & \\
Absence of wrist involvement & $2.05(1.25-3.36)$ & 0.0032 \\
Age at disease onset $<3.6 \mathrm{yrs}$ & $1.81(1.14-2.89)$ & 0.01 \\
\hline
\end{tabular}

* Log-likelihood ratio test. $\mathrm{HR}_{\mathrm{adj}}$ : adjusted hazards ratio.

a poorer functional outcome ${ }^{31}$, and have a lesser likelihood of experiencing a therapeutic response to methotrexate ${ }^{32,33}$. Thus, the presence of arthritis in the wrist joint might identify a subgroup of patients with JIA who deserve earlier introduction of etanercept during their disease course or administration of etanercept in combination with methotrexate. Horneff, et $a l^{34}$ reported a greater frequency of therapeutic response in patients treated with etanercept and methotrexate in combination than in patients who received etanercept monotherapy. Unlike that study, we did not find an association between achievement of inactive disease and concomitant methotrexate administration. However, this observation should be regarded with caution as it was made in the context of a retrospective analysis. The role of methotrexate in enhancing etanercept's effectiveness needs to be examined in a controlled trial.

The only other factor that was associated with inactive disease in Cox regression procedures was an age at disease onset younger than 3.6 years. Younger age at disease presentation was also associated with excellent response to etanercept in the Dutch registry ${ }^{22}$, but was not among the determinants of inactive disease in the German registry ${ }^{21}$. This discrepancy may depend, at least in part, on disparities in patient characteristics, including demographic features, level of disease activity, and distribution of JIA categories. Notably, our patient population had a much younger age at disease onset and included a greater percentage of children with positive ANA status and extended oligoarthritis than the patient samples in the German and Dutch registries.

Several studies, including the 2 registry reports, have shown that anti-TNF agents are less effective in the systemic subset of JIA ${ }^{13,14,21,22,35,36,37,38}$. This phenomenon has been attributed to interleukin 1 (IL-1) and IL-6 playing a greater pathogenetic role than TNF- $\alpha$ in systemic arthritis ${ }^{39,40}$. In accord with these observations, the rate of inactive disease was much lower in our children with systemic arthritis than in those with nonsystemic categories altogether $(29.6 \%$ vs $54.1 \%)$.

We recognize the limitations of the retrospective and noncontrolled design of our study. A retrospective analysis is subject to missing possibly erroneous data. We also acknowledge that the lack of data regarding parent-reported outcomes, namely overall well-being, pain, and functional ability assessments, in a number patients precluded a meaningful analysis of their predictive value. The state of inactive disease was not confirmed with imaging studies. Recently, synovial pathology detected by magnetic resonance imaging or ultrasound, possibly reflecting continuing active disease, has been found in a sizable percentage of children with clinically defined inactive disease $e^{41,42,43}$. We 


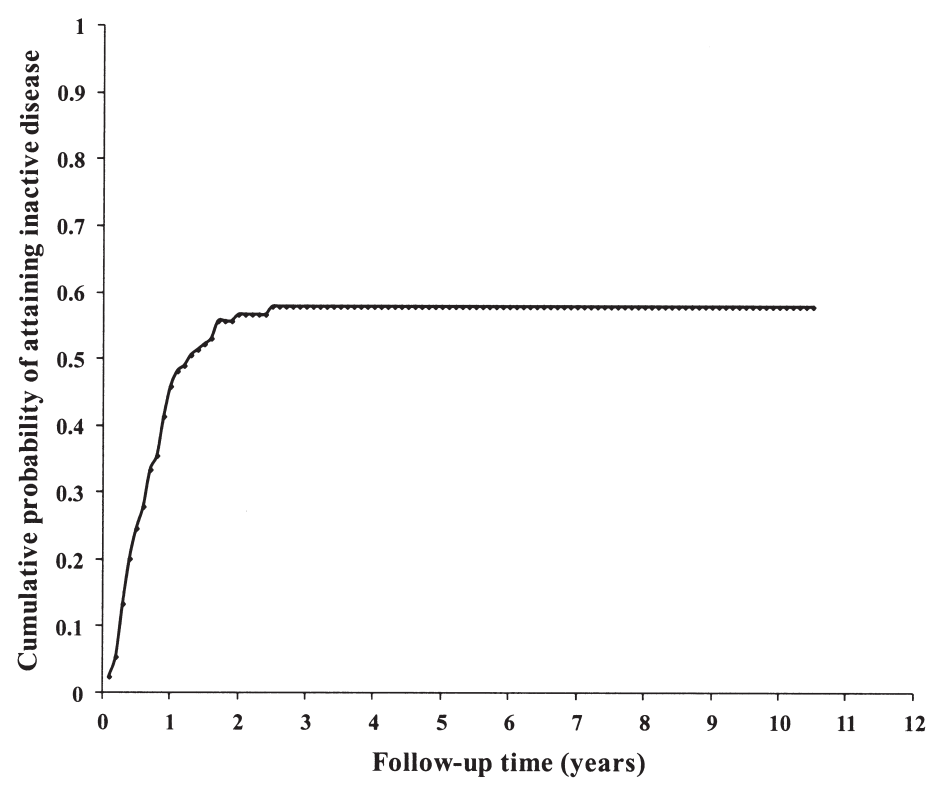

Figure 1. Cumulative probability of attaining state of inactive disease in the entire patient sample; Kaplan-Meier method.

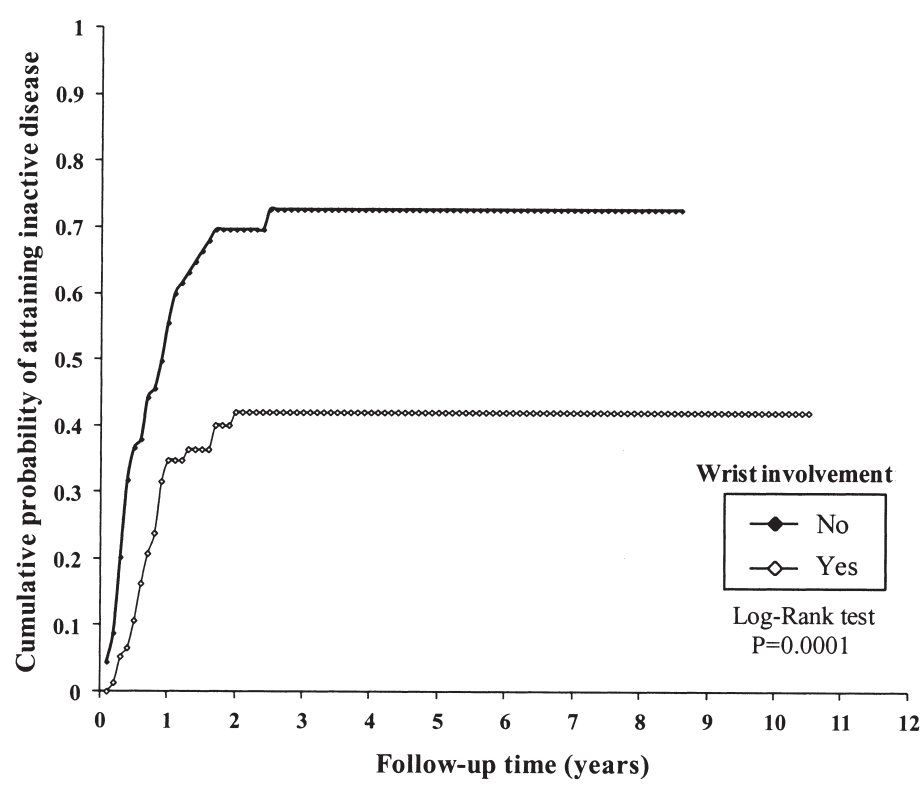

Figure 2. Cumulative probability of attaining state of inactive disease by absence or presence of wrist involvement; Kaplan-Meier method.

did not investigate the rate of disease relapse after discontinuation of etanercept. It is our current policy to continue etanercept at standard dose for 1 year after the occurrence of inactive disease and then to taper it gradually until discontinuation in another year.

Around half of our JIA patients treated with etanercept in standard clinical care were able to achieve the state of inactive disease. Children who lacked wrist involvement and had a younger age at disease onset had a greater likelihood of achieving inactive disease during etanercept administration. Thus, the presence of wrist disease and older age at disease presentation may constitute an indication for earlier introduction of etanercept or its administration in combination with methotrexate. 


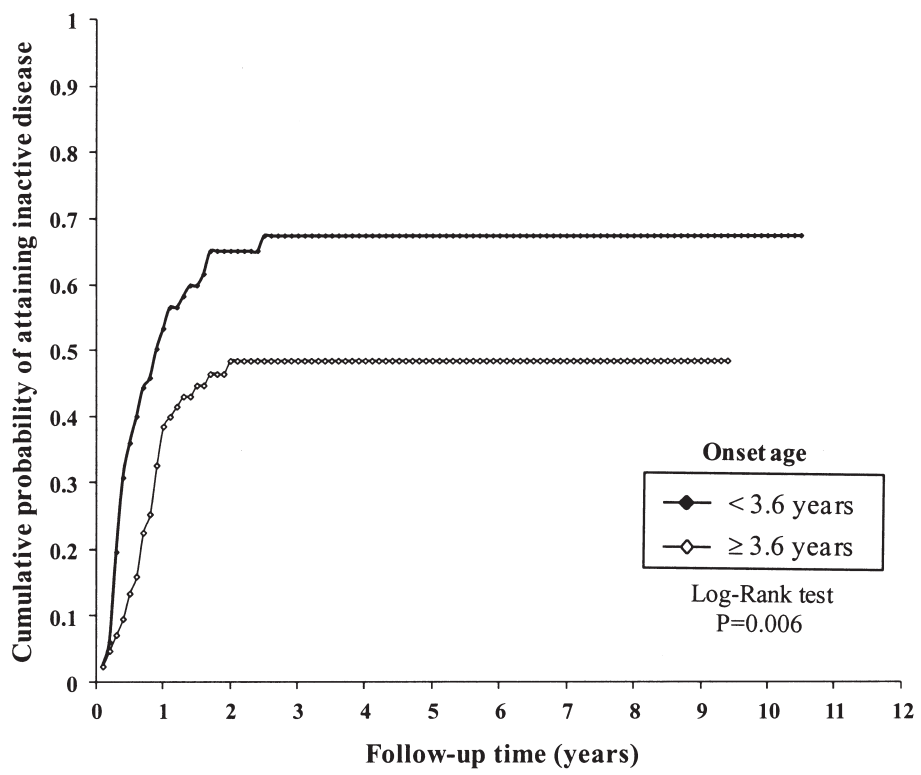

Figure 3. Cumulative probability of attaining state of inactive disease by age at onset $<3.6$ or $\geq 3.6$ years; Kaplan-Meier method.

\section{REFERENCES}

1. Ravelli A, Martini A. Juvenile idiopathic arthritis. Lancet 2007;369:767-78

2. Hashkes PJ, Laxer RM. Medical treatment of juvenile idiopathic arthritis. JAMA 2005;294:1671-84.

3. Hayward K, Wallace CA. Recent developments in anti-rheumatic drugs in pediatrics: Treatment of juvenile idiopathic arthritis. Arthritis Res Ther 2009;11:216.

4. Ruperto N, Martini A. Emerging drugs to treat juvenile idiopathic arthritis. Expert Opin Emerg Drugs 2011;16:493-505.

5. Wallace CA, Ruperto N, Giannini EH. Preliminary criteria for clinical remission for select categories of juvenile idiopathic arthritis. J Rheumatol 2004;31:2290-4.

6. Wallace CA, Giannini EH, Huang B, Itert L, Ruperto N. American College of Rheumatology provisional criteria for defining clinical inactive disease in select categories of juvenile idiopathic arthritis. Arthritis Care Res 2011;63:929-36.

7. Ravelli A, Martini A. Remission in juvenile idiopathic arthritis. Clin Exp Rheumatol 2006;24 Suppl 43:S105-10.

8. Magni-Manzoni S, Ruperto N, Pistorio A, Sala E, Solari N, Palmisani E, et al. Development and validation of a preliminary definition of minimal disease activity in juvenile idiopathic arthritis. Arthritis Rheum 2008;59:1120-7.

9. Martini A, Lovell DJ. Juvenile idiopathic arthritis: State of the art and future perspectives. Ann Rheum Dis 2010;69:1260-3.

10. Ruperto N, Giannini EH, Pistorio A, Brunner HI, Martini A, Lovell DJ. Is it time to move to active comparator trials in juvenile idiopathic arthritis? A review of current study designs. Arthritis Rheum 2010;62:3131-9.

11. Wallace CA, Giannini EH, Spalding SJ, Hashkes PJ, O'Neil KM, Zeft AS, et al. Trial of early aggressive therapy in polyarticular juvenile idiopathic arthritis. Arthritis Rheum 2012;64:2012-21.

12. Lovell DJ, Giannini EH, Reiff A, Cawkwell GD, Silverman ED, Nocton JJ, et al. Etanercept in children with polyarticular juvenile rheumatoid arthritis. Pediatric Rheumatology Collaborative Study Group. N Engl J Med 2000;342:763-9.

13. Quartier P, Taupin P, Bourdeaut F, Lemelle I, Pillet P, Bost M, et al.
Efficacy of etanercept for the treatment of juvenile idiopathic arthritis according to the onset type. Arthritis Rheum 2003; 48:1093-101.

14. Horneff G, Schmeling H, Biedermann T, Foeldvari I, Ganser G, Girschick HJ, et al. The German etanercept registry for treatment of juvenile idiopathic arthritis. Ann Rheum Dis 2004;63:1638-44.

15. Giannini EH, Ilowite NT, Lovell DJ, Wallace CA, Rabinovich CE, Reiff A, et al. Long-term safety and effectiveness of etanercept in children with selected categories of juvenile idiopathic arthritis. Arthritis Rheum 2009;60:2794-804.

16. Halbig M, Horneff G. Improvement of functional ability in children with juvenile idiopathic arthritis by treatment with etanercept. Rheumatol Int 2009;30:229-38.

17. Prince FH, Geerdink LM, Borsboom GJ, Twilt M, van Rossum MA, Hoppenreijs EP, et al. Major improvements in health-related quality of life during the use of etanercept in patients with previously refractory juvenile idiopathic arthritis. Ann Rheum Dis 2010;69:138-42.

18. Giannini EH, Ilowite NT, Lovell DJ, Wallace CA, Rabinovich CE, Reiff A, et al. Effects of long-term etanercept treatment on growth in children with selected categories of juvenile idiopathic arthritis. Arthritis Rheum 2010;62:3259-64.

19. Billiau AD, Loop M, Le PQ, Berthet F, Philippet P, Kasran A, et al. Etanercept improves linear growth and bone mass acquisition in MTX-resistant polyarticular-course juvenile idiopathic arthritis. Rheumatology 2010;49:1550-8.

20. Nielsen S, Ruperto N, Gerloni V, Simonini G, Cortis E, Lepore L, et al. Preliminary evidence that etanercept may reduce radiographic progression in juvenile idiopathic arthritis. Clin Exp Rheumatol 2008;26:688-92.

21. Papsdorf V, Horneff G. Complete control of disease activity and remission induced by treatment with etanercept in juvenile idiopathic arthritis. Rheumatology 2011;50:214-21.

22. Otten MH, Prince FH, Armbrust W, ten Cate R, Hoppenreijs EP, Twilt M, et al. Factors associated with treatment response to etanercept in juvenile idiopathic arthritis. JAMA 2011;306:2340-7.

23. Petty RE, Southwood TR, Manners P, Baum J, Glass DN, 
Goldenberg J, et al. International League of Associations for Rheumatology classification of juvenile idiopathic arthritis: second revision, Edmonton 2001. J Rheumatol 2004;31:390-2.

24. Ravelli A, Varnier GC, Oliveira S, Castell E, Arguedas O, Magnani $\mathrm{A}$, et al. Antinuclear antibody-positive patients should be grouped as a separate category in the classification of juvenile idiopathic arthritis. Arthritis Rheum 2011;63:267-75.

25. Clayton D, Hills M. Statistical models in epidemiology. New York: Oxford University Press; 1993.

26. Magni-Manzoni S, Rossi F, Pistorio A, Temporini F, Viola S, Beluffi G, et al. Prognostic factors for radiographic progression, radiographic damage, and disability in juvenile idiopathic arthritis. Arthritis Rheum 2003;48:3509-17.

27. Cassone R, Falcone A, Rossi F, Magni-Manzoni S, Felici E, Buoncompagni A, et al. Unilateral destructive wrist synovitis in juvenile idiopathic arthritis. Clin Exp Rheumatol 2004;22:637-42.

28. Malattia C, Damasio MB, Magnaguagno F, Pistorio A, Valle M, Martinoli C, et al. Magnetic resonance imaging, ultrasonography, and conventional radiography in the assessment of bone erosions in juvenile idiopathic arthritis. Arthritis Rheum 2008;59:1764-72.

29. Al-Matar MJ, Petty RE, Tucker LB, Malleson PN, Schroeder ML, Cabral DA. The early pattern of joint involvement predicts disease progression in children with oligoarticular (pauciarticular) juvenile rheumatoid arthritis. Arthritis Rheum 2002;46:2708-15.

30. Felici E, Novarini C, Magni-Manzoni S, Pistorio A, Magnani A, Bozzola E, et al. Course of joint disease in patients with antinuclear antibody-positive juvenile idiopathic arthritis. J Rheumatol 2005;32:1805-10.

31. Oen K. Long-term outcomes and predictors of outcomes for patients with juvenile idiopathic arthritis. Best Pract Res Clin Rheumatol 2002;16:347-60.

32. Ravelli A, Viola S, Migliavacca D, Ruperto N, Pistorio A, Martini A, et al. The extended oligoarticular subtype is the best predictor of methotrexate efficacy in juvenile idiopathic arthritis. J Pediatr 1999; 135:316-20.

33. Vilca I, Munitis PG, Pistorio A, Ravelli A, Buoncompagni A, Bica $B$, et al. Pediatric Rheumatology International Trials Organization (PRINTO). Predictors of poor response to methotrexate in polyarticular-course juvenile idiopathic arthritis: Analysis of the PRINTO methotrexate trial. Ann Rheum Dis 2010;69:1479-83.
34. Horneff G, De Bock F, Foeldvari I, Girschick HJ, Michels H, Moebius D, et al. Safety and efficacy of combination of etanercept and methotrexate compared to treatment with etanercept only in patients with juvenile idiopathic arthritis (JIA): Preliminary data from the German JIA Registry. Ann Rheum Dis 2009;68:519-25.

35. Kimura Y, Pinho P, Walco G, Higgins G, Hummell D, Szer I, et al. Etanercept treatment in patients with refractory systemic onset juvenile rheumatoid arthritis. J Rheumatol 2005;32:935-42.

36. Prince FH, Twilt M, ten Cate R, van Rossum MA, Armbrust W, Hoppenreijs EP, et al. Long term follow-up on effectiveness and safety of etanercept in juvenile idiopathic arthritis; the Dutch national register. Ann Rheum Dis 2009;68:635-41.

37. Southwood TR, Foster HE, Davidson JE, Hyrich KL, Cotter CB, Wedderburn LR, et al. British Society for Adolescent and Paediatric Rheumatology Biologics and New Drugs Register. Duration of etanercept treatment and reasons for discontinuation in a cohort of juvenile idiopathic arthritis patients. Rheumatology 2011; 50:189-95.

38. Tynjälä $\mathrm{P}$, Vähäsalo $\mathrm{P}$, Honkanen V, Lahdenne P. Drug survival of the first and second course of antitumour necrosis factor agents in juvenile idiopathic arthritis. Ann Rheum Dis 2009;68:552-7.

39. Pascual V, Allantaz F, Arce E, Punaro M, Banchereau J. Role of interleukin-1 (IL-1) in the pathogenesis of systemic onset juvenile idiopathic arthritis and clinical response to IL-1 blockade. J Exp Med 2005;201:1479-86.

40. De Benedetti F, Martini A. Is systemic juvenile rheumatoid arthritis an interleukin 6 mediated disease? J Rheumatol 1998;25:929-31.

41. Tzaribachev N, Fritz J, Horger M. Silent arthritis in JIA children with clinically inactive disease detected by MRI. Ann Rheum Dis 2011;70 Suppl 3:90.

42. Rebollo-Polo M, Koujok K, Weisser C, Jurencak R, Bruns A, Roth $\mathrm{J}$, et al. Ultrasound findings on patients with juvenile idiopathic arthritis in clinical remission. Arthritis Care Res 2011;63:1013-9.

43. Magni-Manzoni S, Scirè CA, Ravelli A, Klersy C, Rossi S, Muratore V, et al. Ultrasound-detected synovial abnormalities are frequent in clinically inactive juvenile idiopathic arthritis, but do not predict a flare of synovitis. Ann Rheum Dis 2012 June 26 [E-pub ahead of print]. 